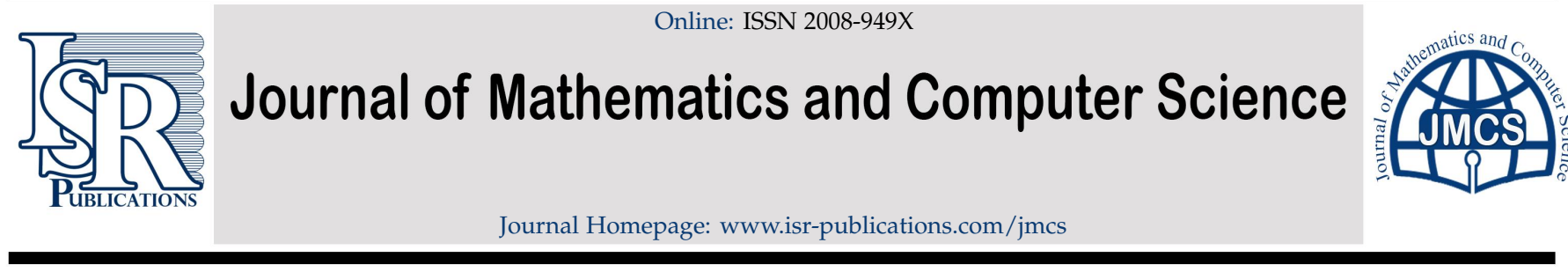

\title{
On the prime fuzzy m-bi ideals in semigroups
}

\author{
Mohammad Munir $^{\mathrm{a}}$, Nasreen Kausar ${ }^{\mathrm{b}, *}$, Salahuddin $^{\mathrm{c}}$, Tehreem $^{\mathrm{d}}$ \\ ${ }^{a}$ Government Postgraduate College, Abbottabad, Pakistan. \\ ${ }^{b}$ Department of Mathematics, University of Agriculture, Faisalabad, Pakistan. \\ ${ }^{c}$ Department of Mathematics, Jazan University, Jazan, Kingdom of Saudi Arabia. \\ ${ }^{d}$ Department of Mathematics, Air University (AU), Islamabad, Pakistan.
}

\begin{abstract}
In this article, we build up the ideas of the fuzzy strongly prime, prime and semiprime m-bi ideals in a semigroup. We additionally investigate the conditions that when the fuzzy $\mathrm{m}$-bi ideals are irreducible and strongly irreducible. We have demonstrated that a semigroup is m-regular and $m$-intraregular if and only if its very fuzzy m-bi ideal is a semiprime, likewise a subset of a semigroup is its $m$-bi ideal if and only if the characteristic function on this subset is a fuzzy $m$-bi ideal.
\end{abstract}

Keywords: Fuzzy m-bi ideals, strongly prime m-bi ideals, semiprime m-bi ideals, bipotency.

2020 MSC: 20M35, 20M99.

(C)2020 All rights reserved.

\section{Introduction and essential concepts}

A non-void set $M$ together with a given associative binary operation - is known as a semigroup. Semigroups are basically a generalization of groups originated in the early twentieth century. These, due to their simplest structures amongst all the algebraic structures, have been in use in all the branches of the science, technologies and mathematics since their discovery. The books cited as $[2,3]$ study completely the semigroups and their applications in all fields. Any function $\mu: M \longrightarrow[0,1]$ is known as fuzzy subset of $M$ [14]. The study of fuzzy sub-structures like subsemigroups and fuzzy sets is unique, among the branches of mathematics, in the sense that it is capable to handle the numerical as well as linguistic knowledge [9]. It models the complex systems of decision making, managerial tasks and huge networks. It can handle uncertainties, non-linear and distributive processes [11]. For complete study of the fuzzification of semigroups and their uses and applications, the books mentioned in References $[8,9,15]$ are worthy to be referred here.

Kausar et al. [13], discussed the non-associative ordered semigroups by their fuzzy bi-ideals. Kausar [4], established the ordered AG-groupoids by the properties of fuzzy ideals with thresholds $(\alpha, \beta]$. Salahuddin et al. [5], studied the anti fuzzy interior ideals on ordered AG-groupoids. Munir et al. [6], explored the

\footnotetext{
*Corresponding author

Email addresses: dr.mohammadmunir@gmail.com (Mohammad Munir), kausar.nasreen57@gmail.com (Nasreen Kausar)

doi: $10.22436 /$ jmcs.021.04.08
}

Received: 2020-02-27 Revised: 2020-03-31 Accepted: 2020-04-20 
intuitionistic fuzzy bi-ideals in ordered AG-groupoids and also characterized the ordered AG-groupoids in terms of intuitionistic fuzzy bi-ideals.

In order to develop the theory of these fuzzy m-bi ideals, we first define the essential concepts which will be used later. Throughout our course of work, the letter $M$ is understood to be a semigroup.

Consider the fuzzy subsets $\lambda$ and $\mu$ of a semigroup $M$, the proposition $\lambda \leqslant \mu$ implies that $\lambda(x) \leqslant \mu(x)$, $\forall x \in M$ [8]. The conjunction (disjunction) of $\mu$ and $\nu$ is defined by $\mu \vee v(x)=\max [\mu(x), \nu(x)](\mu \wedge \nu(x)=$ $\min [\mu(x), v(x)])$. Their composition, denoted by $\mu \circ \nu$, is defined by

$$
f \circ g(z)= \begin{cases}\sup _{z=x y, x, y \in M}\{\min [f(x), g(y)]\}, & \text { if } z \text { is representable as } z=x y, \\ 0, & \text { otherwise, }\end{cases}
$$

for all $z \in M . \mu \vee v, \mu \wedge v$ and $\mu \circ v$ are fuzzy sets of $M$. The characteristic function of $A \subseteq M$ defined by

$$
\chi_{A}(t)= \begin{cases}1, & \text { if } t \in A, \\ 0, & \text { if } t \notin A,\end{cases}
$$

is a fuzzy subset of $M$.

The $\circ$ has precedence over $\vee$ and $\wedge . \mu$ is called a fuzzy subsemigroup of $M$ if $\mu \circ \mu \leqslant \mu$. The fuzzy subsemigroup $\mu$ is called a fuzzy left respectively fuzzy right ideal of $M$ if $\chi_{M} \circ \mu \leqslant \mu$, respectively $\mu \leqslant \chi_{M} \circ \mu . \mu$ is a fuzzy ideal of $M$ if $\chi_{M} \circ \mu \circ \chi_{M} \leqslant \mu . \mu$ is a fuzzy bi-ideal of $M$ if $\mu \circ \chi_{M} \circ \mu \leqslant \mu$. $\mu$ is known as a fuzzy quasi-ideal of $M$ if $\mu \circ \chi_{M} \cap \chi_{M} \circ \mu \leqslant \mu$ [1]. A fuzzy subset $\mu$ of a semigroup $M$ is called fuzzy $m$-bi ideal (see [10]) if $\mu \circ \chi_{M^{m}} \circ \mu \leqslant \mu$, where

$$
\chi_{M^{m}}(t)= \begin{cases}1, & \text { if } t \in M^{m}, \\ 0, & \text { if } t \notin M^{m},\end{cases}
$$

and $m$ is a positive integer called the bipotency of the fuzzy $m$-bi ideal of $M$. A fuzzy subset $\mu$ of a semigroup $M$ is called fuzzy $m$-quasi ideal if $\mu \circ \chi_{M^{m}} \wedge \chi_{M^{m}} \circ \mu \leqslant \mu, m$ is a positive integer. Every subset of $M^{m}$ is called the $m$-subset of $M$. Every subsemigroup of $M^{m}$ is called $m$-subsemigroup of $M$.

Definition 1.1. Let $a \in M$ and $t \in(0,1\}$, then the fuzzy subset of $M$, defined by

$$
a_{t}(x)= \begin{cases}t, & \text { if } x=a, \forall x \in M^{m} \\ 0, & \text { otherwise }\end{cases}
$$

is called a fuzzy m-point of $M$.

Definition 1.2. Let $A \subseteq M$ and $t \in(0,1]$, then the fuzzy subset of $M$, defined by

$$
A_{t}(x)= \begin{cases}t, & \text { if } x \in A, \forall x \in M^{m} \\ 0, & \text { otherwise }\end{cases}
$$

is called a fuzzy m-subset of $M$.

The fuzzy m-subset $A$ of $M$ is the union of the fuzzy m-points a of $M$.

In the accompanying sections, we characterize the prime fuzzy $\mathrm{m}$-bi and their related classes. We investigate their significant properties, and portray the m-regular and m-intraregular semigroups by their properties. 


\section{Prime fuzzy m-Bi ideals}

In [12], Shabir et al. presented the concepts of the prime fuzzy bi ideals along with other associated fuzzy bi ideals in the simgroups, we present these ideas for the case of $m$-bi ideals and demonstrate their important properties in this section.

Definition 2.1. A fuzzy $m$-bi ideal, $\mu$, of $M$ is termed as its prime(strongly prime) fuzzy $m$-bi ideal if for any fuzzy m-bi ideals $\lambda$, $v$ of $M$, the proposition " $\lambda \circ \nu \leqslant \mu(\lambda \circ \nu \wedge \nu \circ \lambda \leqslant \mu)$ " implies " $\lambda \leqslant \mu$ or $v \leqslant \mu$ ".

Definition 2.2. A fuzzy m-bi best $\mu$ of $M$ is known as its semiprime fuzzy $m$-bi ideal if for any fuzzy m-bi ideal $\lambda$ of $M, \lambda \circ \lambda \leqslant \mu$ implies $\lambda \leqslant \mu$.

Strongly prime fuzzy $m$-bi ideal $\Rightarrow$ Prime fuzzy $m$-bi ideal $\Rightarrow$ Semiprime fuzzy $m$-bi ideal; however, the converses do not follow. This is discussed in Example 3.7.

Example 2.3. The characteristic functions $\chi_{M}$ and $\chi_{M^{m}}, m$ - a positive, are prime fuzzy $m$-bi ideal of $M$. Additionally, these two are also semiprime and strongly prime fuzzy m-bi ideals.

Definition 2.4. Fuzzy m-bi ideal $\mu$ of $M$ is said to be an irreducible (strongly irreducible) fuzzy m-bi ideal if for any two fuzzy m-bi ideals $\lambda$ and $\nu$ of $M$, the proposition $\lambda \wedge \nu=\mu(\lambda \wedge \nu \leqslant \mu)$ implies either $\lambda=\mu$ or $v=\mu(\lambda \leqslant \mu$ or $v \leqslant \mu)$.

Definition 2.5. If the fuzzy m-bi ideal $\lambda$ satisfies the condition " $\lambda \circ \lambda=\lambda$ ", then $\lambda$ is known as idempotent.

Strongly irreducible $\mathrm{m}$-bi ideal $\Rightarrow$ irreducible $\mathrm{m}$-bi ideal, but not the converse. See the following example.

Theorem 2.6. The characteristic function $\chi_{\mathrm{I}}$ on a non-empty subset I of $\mathrm{M}$ is a prime fuzzy $\mathrm{m}$-bi ideal iff I is prime $\mathrm{m}$-bi ideal of $\mathrm{M}$.

Proof. Let I be prime $m$-bi ideal of $M$. We want to show that $\chi_{I}$ is a prime fuzzy $m$-bi ideal of $M$. For it, let $\mu$ and $\lambda$ be fuzzy m-bi ideals of $M$ with the property that $\mu \circ \lambda \leqslant \chi_{I}$. If $\mu \not \leq \chi_{I}$, then $\exists$ a fuzzy m-point $x_{t} \leqslant \mu(t>0)$ such that $x_{t} \not \leq x_{I}$. For any $y_{r} \leqslant \lambda(r \neq 0)$, since

$$
\mathrm{B}\left(\mathrm{x}_{\mathrm{t}}\right) \circ \mathrm{B}\left(\mathrm{y}_{\mathrm{r}}\right) \leqslant \mu \circ \lambda \leqslant x_{\mathrm{I}},
$$

we have, for all $z \in M^{m}$,

$$
B\left(x_{t}\right) \circ B\left(y_{r}\right)(z)= \begin{cases}t \wedge r>0, & \text { if } z \in B(x) \cdot B(y), \\ 0, & \text { otherwise. }\end{cases}
$$

We get from (2.1) and (2.1) above, $\mathrm{B}(\mathrm{x}) \cdot \mathrm{B}(\mathrm{y}) \subseteq \mathrm{I}$. I being prime gives $\mathrm{B}(\mathrm{x}) \subseteq \mathrm{I}$ or $\mathrm{B}(\mathrm{y}) \subseteq \mathrm{I}$. Since $\mathrm{x}_{\mathrm{t}} \notin \mathrm{x}_{\mathrm{I}}$, we have $\lambda=\vee_{y_{r} \leqslant \lambda y_{r}} \leqslant \chi_{I}$. To prove the converse, let $A B \subseteq I$ for $m$-bi ideals $A, B$ in $M$. As $\chi_{A}, \chi_{B}$ are fuzzy $m$-bi ideals of $M$ and $\chi_{A} \circ \chi_{B}=\chi_{A B} \leqslant \chi_{I}$ [12]. From hypothesis, we have $\chi_{A} \leqslant \chi_{I}$ or $\chi_{B} \leqslant \chi_{I}$. Thus $A \subseteq$ I or $B \subseteq$ I making I a fuzzy prime m-bi ideal of $M$.

Theorem 2.7. A nonempty subset I of $\mathrm{M}$ is strongly prime $\mathrm{m}$-bi ideal iff the characteristic function $\mathrm{\chi}_{\mathrm{I}}$ is strongly prime fuzzy m-bi ideal.

Proof. Let $I$ is strongly prime $m$-bi ideal of $M$. Then $\chi_{I}$ is fuzzy $m$-bi ideal of $M$. To show that $\chi_{I}$ is strongly prime, we take $\mu \circ \lambda \wedge \lambda \circ \mu \leqslant \chi_{I}$ for fuzzy m-bi ideals $\mu$ and $\lambda$. If $\mu \not \leq C_{I}, \exists$ a fuzzy m-point $x_{t} \leqslant \mu(t>0)$ such that $x_{t} \not x_{I}$. Now for any $y_{r} \leqslant \lambda(r \neq 0)$, we have

$$
B\left(x_{t}\right) \circ B\left(y_{r}\right) \wedge B\left(y_{r}\right) \circ B\left(x_{t}\right) \leqslant \mu \circ \lambda \wedge \lambda \circ \mu \leqslant x_{I} .
$$


Next, $\forall z \in M^{m}$, we have

$$
\left(B\left(x_{t}\right) \circ B\left(y_{r}\right) \wedge B\left(y_{r}\right) \circ B\left(x_{t}\right)\right)(z)= \begin{cases}t \wedge r, & \text { if } z \in B(x) B(y) \cap B(y) B(x), \\ 0, & \text { otherwise. }\end{cases}
$$

From (2.2) and (2.3), $\mathrm{B}(\mathrm{x}) \mathrm{B}(\mathrm{y}) \cap \mathrm{B}(\mathrm{y}) \mathrm{B}(\mathrm{x}) \subseteq \mathrm{I}$. I being strongly prime implies that $\mathrm{B}(\mathrm{x}) \subseteq \mathrm{I}$ or $\mathrm{B}(\mathrm{y}) \subseteq \mathrm{I}$. Since $x_{t} \not \leq x_{I}, B(x) \nsubseteq I$. Consequently, $B(y) \subseteq$ I bringing $y_{r} \leqslant x_{I}$. Thus, $\lambda=\underset{y_{r} \leqslant g}{V} y_{r} \leqslant x_{I}$. For the converse, if $A$ and $B$ are fuzzy m-bi ideals of $M$ such $A B \cap B A \subseteq I$, then $\chi_{A}, \chi_{B}$ are fuzzy m-bi ideals of $M$ and $\chi_{A} \circ \chi_{B} \wedge \chi_{B} \circ \chi_{A}=\chi_{A B \cap B A} \leqslant \chi_{I}$. From hypothesis, we have $\chi_{A} \leqslant \chi_{I}$ or $\chi_{B} \leqslant C_{I}$. Eventually, either $A \subseteq$ I or $B \subseteq I$.

Theorem 2.8. $A \emptyset \neq A \subseteq M$ is a semiprime $m$-bi ideal of $M$ iff $\chi_{A}$ is a semiprime fuzzy $m$-bi ideal of $M$.

Proof. Suppose that $\chi_{A}$ is a semiprime fuzzy $m$-bi ideal of $M$, then $A$ is a m-bi ideal of $M$. Let $B$ be an $m$-bi ideal in $M$ with the condition hat $B^{2} \subseteq A$. That is, $\chi_{B} \circ \chi_{B}=\chi_{B} \leqslant \chi_{A}$. Since $\chi_{A}$ is semiprime, $\chi_{\mathrm{B}} \leqslant \chi_{\mathrm{A}}$, that is $\mathrm{B} \subseteq \mathrm{I}$.

Conversely, suppose that $A$ is a semiprime $m$-bi ideal of $M$, and $\lambda$ is a fuzzy $m$-bi ideal of $M$ with the property that $\lambda \circ \lambda \leqslant \chi_{A}$. If $\lambda \not \leq \chi_{A}$, then $\exists$ an m-point $x_{t} \leqslant \lambda(t>0)$ such that $x_{t} \not \leq \chi_{A}$. Now since, $\mathrm{B}\left(x_{\mathrm{t}}\right) \circ \mathrm{B}\left(\mathrm{x}_{\mathrm{t}}\right) \leqslant \lambda \circ \lambda \leqslant x_{\mathrm{I}}$, therefore, $\forall, z \in M$, we have

$$
\mathrm{B}\left(x_{\mathrm{t}}\right) \circ \mathrm{B}\left(x_{\mathrm{t}}\right)(z)= \begin{cases}\mathrm{t}, & \text { for } z \in \mathrm{B}(\mathrm{x}) \mathrm{B}(\mathrm{x}), \\ 0, & \text { otherwise. }\end{cases}
$$

Thus, $\mathrm{B}(x) \mathrm{B}(x) \subseteq \mathrm{A}$. This implies that $\mathrm{B}(x) \subseteq A$; because $A$ being semiprime. Hence, $\mathrm{B}\left(x_{t}\right) \leqslant \chi_{A}$. But $x_{t} \leqslant B\left(x_{t}\right)$ so $x_{t} \leqslant x_{A} ;$ a contradiction. Hence $\lambda \leqslant x_{I}$.

Lemma 2.9. If $\left\{\mu_{i}: i \in I\right\}$ is a family of prime fuzzy $m$-bi ideals of $M$, then the intersection $\mathcal{A}=\bigwedge_{i \in \mathrm{I}} \mu_{i}$ is a semiprime fuzzy $\mathrm{m}$-bi ideal of $\mathrm{M}$.

Proof. $\mathcal{A}$ is clearly a fuzzy $m$-bi ideal of $M$. Let $\delta$ be fuzzy m-bi ideal with the property that $\delta \circ \delta \leqslant \mathcal{A}$. This implies $\delta \circ \delta \leqslant \bigwedge_{i \in \mathrm{I}} \mu_{i}$. Then $\delta \circ \delta \leqslant \mu_{i} \forall i \in I$. Since each $\mu_{i}$ is a fuzzy prime $m$-bi ideal of $M$, so $\delta \leqslant \mu_{i} \forall i \in I$. Hence $\delta \leqslant \bigwedge_{i \in I} \mu_{i}=\mathcal{A}$. Thus $\mathcal{A}$ is fuzzy semiprime m-bi ideal of $M$.

Corollary 2.10. The intersection of the fuzzy prime(strongly prime) $\mathrm{m}$-bi ideals of $\mathrm{M}$ is fuzzy prime (strongly prime) $\mathrm{m}$-bi ideal of $\mathrm{M}$.

Proposition 2.11. Strongly irreducible semiprime fuzzy $\mathrm{m}$-bi coincides with the strongly prime fuzzy m-bi ideal in a semigroup $M$.

Proof. If $\mu$ is supposed to be semiprime fuzzy m-bi ideal of $M$ which is also strongly irreducible, and $\lambda$ and $v$ are fuzzy $m$-bi ideals of $M$ with the condition $\lambda \circ v \wedge v \circ \lambda \leqslant \mu$. As $(\lambda \wedge v)^{2} \leqslant \lambda \circ v$ and $(\lambda \wedge v)^{2} \leqslant v \circ \lambda$. Thus $(\lambda \wedge v)^{2} \leqslant \lambda \circ \nu \wedge v \circ \lambda$ and so $(\lambda \wedge v)^{2} \leqslant \mu$. By our hypothesis, $\lambda \wedge v \leqslant \mu ; \mu$ being semiprime. Again by our hypothesis, either $\lambda \leqslant \mu$ or $v \leqslant \mu ; \mu$ being strongly irreducible. Thus $\mu$ is strongly prime fuzzy m-bi ideal.

\section{Characterizing $m$-regular and $m$-intraregular semigroups}

A detail study of the m-regular and m-intraregular semigroups is presented in [10]. In the following section, we study the semigroups in which each fuzzy m-bi ideal is semiprime or strongly prime.

Proposition 3.1. Corresponding to a fuzzy $m$-bi ideal $\mu$ of $M$ satisfying $\mu(a)=\alpha$, for $a \in M^{m}$ and $\alpha \in(0,1], \exists$ an irreducible fuzzy $m$-bi ideal $\lambda$ of $M$ such that $\mu \leqslant \lambda$ and $\lambda(a)=\alpha$. 
Proof. Suppose that $\mathcal{C}$ is the collection of all fuzzy $m$-bi ideal $v$ of $M$ such that $v(a)=\alpha$ and $\mu \leqslant v$. $\mathcal{C} \neq \emptyset$ because $\mu \in X$. $\mathcal{C}$ is clearly a partially ordered set with respect to inclusion of sets. If $\mathcal{D}=\left\{h_{i}: i \in I\right\}$ is a totally ordered subset of $\mathcal{C}$, then $\underset{i \in I}{\vee} h_{i}$ is a fuzzy m-bi ideal of $M$ containing $\mu$. This is because, if $a, b \in$ $M$ and $x \in M^{m}$ then, $\left.\left(\bigvee_{i \in I} h_{i}\right)(a \times b)=\bigvee_{i \in I}\left(h_{i}(a x b)\right) \geqslant \bigvee_{i \in I}\left(h_{i}(a)\right) \wedge h_{i}(b)\right)=\left(\bigvee_{i \in I} h_{i}(a)\right) \wedge\left(\bigvee_{i \in I}\left(h_{i}(b)\right)=\right.$ $\left(\vee_{i \in I} h_{i}\right)(a) \wedge\left(\underset{i \in I}{\vee} h_{i}\right)(b)$. Hence, $\vee_{i \in I} h_{i}$ is a fuzzy m-bi ideal of $M$. As $\mu \leqslant h_{i}$ for each $i \in I$, so $\mu \leqslant \vee_{i \in I} h_{i}$. Also, $\left(\underset{i \in I}{\vee} h_{i}\right)(a)=\underset{i \in I}{\vee} h_{i}(a)=\alpha$. Thus, $\underset{i \in I}{\vee} h_{i}$ is the supremum of $\mathcal{D}$. By Zorn's Lemma, $\exists$ a maximal fuzzy $m$-bi ideal $\lambda$ of $M$ such that $\mu \leqslant \lambda$ and $\lambda(a)=\alpha$. It is left only to show that $\lambda$ is an irreducible fuzzy $m$-bi ideal of $M$. Let $\lambda=\lambda_{1} \wedge \lambda_{2}$ where $\lambda_{1}$ and $\lambda_{2}$ are fuzzy $m$-bi ideal of $M$. We have to show that $\lambda=\lambda_{1}$ or $\lambda=\lambda_{2}$. Suppose on the contrary that, $\lambda \neq \lambda_{1}$ and $\lambda \neq \lambda_{2}$. Since $\lambda(a)=\alpha$ it implies that $\lambda_{1}(a) \neq \alpha$ and $\lambda_{2}(a) \neq \alpha$. Hence $\alpha=\lambda(a)=\left(\lambda_{1} \wedge \lambda_{2}\right)(a) \neq \alpha$; a contradiction. Hence our supposition is wrong, so either $\lambda=\lambda_{1}$ or $\lambda=\lambda_{2}$. Thus $\lambda$ is an irreducible fuzzy $m$-bi ideal of $M$.

The following theorem describes the semigroups in which each fuzzy m-bi ideal is semiprime.

Theorem 3.2 ([7]). The following assertions are equivalent for a semigroup $M$.

1. $M$ is both $\mathrm{m}$-regular and $\mathrm{m}$-intraregular.

2. $\mu \circ \mu=\mu$ for every fuzzy m-bi ideal $\mu$ of $M$.

3. $\lambda \wedge v=\lambda \circ \vee \wedge v \circ \lambda$ for all fuzzy $m$-bi ideals $\lambda$ and $v$ of $M$.

4. Each fuzzy $m$-bi ideal of $M$ is semiprime.

5. Each proper fuzzy $\mathrm{m}$-bi ideal of $\mathrm{M}$ is the intersection of irreducible semiprime fuzzy $\mathrm{m}$-bi ideals of $\mathrm{M}$ which contain it.

Proof.

$(1) \Rightarrow(2)$ : Clearly $\mu \circ \mu \leqslant \mu$. Let a be an element of $M$, and since $M$ is both m-regular and m-intraregular, so there are $u, v, w \in M^{m}$ so that $a=a u a$ and $a=v a^{2} w$. We have $a=($ auva)(awua). Hence, $\left.\mu \circ \mu(a)=\sup _{a=b c}\{\min \{\mu(b), \mu(c)\}\} \geqslant \sup _{a=b c}\{\min \{\mu(a u v a), \mu(a w u a)\}\}=\min _{a=b c}\{\mu(b), \mu(c)\}\right\}=\mu \wedge \mu(a)=$ $\mu(a)$. That is, $\mu \circ \mu(a) \geqslant \mu(a)$. Thus, $\mu \circ \mu=\mu$.

(2) $\Rightarrow$ (3): By our hypothesis, $\lambda \wedge v=\lambda \wedge v \circ \lambda \wedge v=(\lambda \wedge v \circ \lambda) \wedge(\lambda \wedge v \circ v)=\lambda \circ v \wedge v \circ \lambda$, as required.

(3) $\Rightarrow$ (4): Let $\mu, \lambda$ be fuzzy m-bi ideals of $M$ such that $\mu \leqslant \lambda$. By hypothesis, $\mu=\mu \wedge \mu=\mu \circ \mu \wedge \mu \circ \mu=$ $\mu \circ \mu=\mu^{2}$. Thus $\mu \leqslant \lambda$. Hence every fuzzy $m$-bi ideal of $M$ is semiprime.

(4) $\Rightarrow$ (5): Suppose that $\mathcal{C}=\left\{\mu_{i}: i \in I\right\}$ is the set of all irreducible fuzzy m-bi ideals of $M$ which contain a proper fuzzy m-bi ideal $\mu$ of $M$. $\mathcal{C} \neq \emptyset$ because $\mu \in \mathcal{C}$. Moreover, $\mu \leqslant \wedge_{i \in I} \mu_{i}$. For, $a \in M$, by Proposition 3.1, $\exists$ an irreducible fuzzy $m$-bi ideal $\mu$ of $M$ such that $\mu \leqslant \mu$ and $\mu(a)=\mu(a)$. Therefore, $\mu \in\left\{\mu_{i}: i \in I\right\}$. Hence $\bigwedge_{i \in I} \mu_{i} \leqslant \mu$. So, $\bigwedge_{i \in I} \mu_{i}(a) \leqslant \mu_{\alpha}(a)=\mu(a)$. By hypothesis, each fuzzy $m$-bi ideal of $M$ is semiprime. So each fuzzy $m$-bi ideal of $M$ is the intersection of all irreducible semiprime fuzzy $m$-bi ideals of $M$ which contain it.

$(5) \Rightarrow(2)$ : Let $\mu$ be a fuzzy $m$-bi ideal of $M$, this implies $\mu^{2}$ is a fuzzy $m$-bi ideal of $M$ as well. $\mu$ contains $\mu^{2}$. By our hypothesis $\mu^{2}=\bigwedge_{i \in I} \mu_{i}$, where each $\mu_{i}$ is irreducible semiprime fuzzy $m$-bi ideal of $M$. So $\mu^{2} \leqslant \mu_{i} \forall i \in I$. Thus $\mu \leqslant \mu_{i} \forall i \in I$, since $\mu_{i}$ are semiprime. Consequently, $\mu \leqslant \bigwedge_{i \in I} \mu_{i}=\mu^{2}$, so that $\mu^{2}=\mu$.

Proposition 3.3 ([12]). Let $M$ be a m-regular and m-intraregular semigroup. Then the following assertions for a fuzzy $\mathrm{m}$-bi ideal $\mu$ of $\mathrm{M}$ are equivalent.

1. $\mu$ is strongly irreducible $m$-bi ideal.

2. $\mu$ is strongly prime m-bi ideal. 
Proof. (1) $\Rightarrow$ (2): Let $\mu$ is strongly irreducible m-bi ideal of $M$ with the property that $\lambda \circ v \wedge v \circ \lambda \leqslant \mu$, for any two fuzzy m-bi ideals $\lambda$ and $\nu$ of $M$. Theorem 3.2 (3) gives $\lambda \wedge v \leqslant \mu$. By our hypothesis, $\lambda \leqslant \mu$ or $v \leqslant \mu$ making $\mu$ a strongly prime m-bi ideal of $M .(2) \Rightarrow(1)$ is similar.

Theorem 3.4 ([12]). The following conditions for a semigroup $M$ are equivalent.

1. $M$ is both $m$-regular and $m$-intraregular and the collection of fuzzy $m$-bi ideals of $M$ is totally ordered by set inclusion.

2. Every fuzzy $\mathrm{m}$-bi ideal of $\mathrm{M}$ is strongly prime.

Proof.

$(1) \Rightarrow(2)$ : Let the hypothesis (1) holds. It is required to show that an arbitrary fuzzy m-bi ideals $\mu$ of $M$ is strongly prime. Let $\lambda$ and $v$ are two fuzzy $m$-bi ideals of $M$ satisfying the property that $\lambda \circ v \wedge v \circ \lambda \leqslant \mu$. Using our hypothesis in lieu with Theorem 3.2, we have $\lambda \circ v \wedge \nu \circ \lambda=\lambda \wedge \nu$. This implies that $\lambda \wedge v \leqslant \mu$. But the collection of fuzzy m-bi ideals of $M$ is totally ordered, so either $\lambda \leqslant v$ or $v \leqslant \lambda$. Consequently, either $\lambda \leqslant \mu$ or $v \leqslant \mu$ making $\mu$ strongly prime.

$(2) \Rightarrow(1)$ : Suppose the validity of the proposition (2) holds. Since a strongly prime fuzzy m-bi ideals is semiprime, so every fuzzy m-bi ideal of $M$ is semiprime. So by Theorem 3.2, $M$ comes off to be both $m$-regular and m-intraregular. The set of fuzzy $m$-bi ideals of $M$ is to be shown totally ordered. For this purpose, let $\lambda$ and $v$ be arbitrary two fuzzy m-bi ideals of $M$. Theorem 3.2 brings $\lambda \wedge v=(\lambda \circ v) \wedge(\nu \circ \lambda)$. Since every fuzzy m-bi ideals is strongly prime, so $\lambda \wedge v$ is strongly prime. This implies either $\lambda \leqslant \lambda \wedge v$ or $v \leqslant \lambda \wedge v$. If $\lambda \leqslant \lambda \wedge v$, then $\lambda \leqslant v$ and if $v \leqslant \lambda \wedge v$, then $v \leqslant \lambda$. This marks the end of the proof.

Theorem 3.5 ([12]). Given a semigroup $M$, with the collection of its fuzzy m-bi ideals totally ordered under set inclusion, the following given assertions are equivalent.

1. Every fuzzy $\mathrm{m}$-bi ideals of $\mathrm{M}$ is prime.

2. $M$ is $\mathrm{m}$-regular and $\mathrm{m}$-intraregular.

Proof.

$(1) \Rightarrow(2)$ : Assume every fuzzy $m$-bi ideals of $M$ is prime; and so is semiprime obviously. By Theorem $3.2, M$ is both $\mathrm{m}$-regular and $\mathrm{m}$-intraregular.

$(2) \Rightarrow(1)$ : Suppose $\mu$ be any fuzzy $m$-bi ideals of $M$, which is both $m$-regular and $m$-intraregular, such that $\lambda \circ v \leqslant \mu$ for $m$-bi ideals $\lambda$ and $v$ of $M$. Since the fuzzy m-bi ideals of $M$ makes a totally ordered set, so either $\lambda \leqslant v$ or $v \leqslant \lambda$. If we take $\lambda \leqslant v$, then $\lambda \circ \lambda \leqslant \lambda \circ v \leqslant \mu$. By Theorem 3.2, $\mu$ is semiprime, so $\lambda \leqslant \mu$; making $\mu$ a prime fuzzy $m$-bi ideals of $M$.

Theorem 3.6 ([12]). For a semigroup $\mathrm{M}$, the following assertions are equivalent.

1. The set of fuzzy $\mathrm{m}$-bi ideals of $\mathrm{M}$ is totally ordered under inclusion.

2. Each fuzzy $\mathrm{m}$-bi ideals of $\mathrm{M}$ is strongly irreducible.

3. Each fuzzy $\mathrm{m}$-bi ideals of $\mathrm{M}$ is irreducible.

Proof.

$(1) \Rightarrow(2)$ : Let $\mu$ be an arbitrary fuzzy $m$-bi ideals of $M$ and $\lambda, v$ are two fuzzy $m$-bi ideals of $M$ such that $\lambda \wedge v \leqslant \mu$. Since the fuzzy $m$-bi ideals of $M$ makes a totally ordered, so either $\lambda \leqslant v$ or $v \leqslant \lambda$. This gives $\lambda \wedge v=v$ or $\lambda \wedge v=\lambda$. Hence $\lambda \wedge v \leqslant \mu$ results in either $v \leqslant \mu$ or $\lambda \leqslant \mu$. Eventually, $\mu$ is strongly irreducible.

(2) $\Rightarrow$ (3): is trivial. 
(3) $\Rightarrow(1)$ : Let $\lambda, v$ be any two fuzzy $m$-bi ideals of $M$. Then $\lambda \wedge v$ is a fuzzy $m$-bi ideals of $M$. Also $\lambda \wedge v=\lambda \wedge v$. So by hypothesis, either $\lambda=\lambda \wedge v$ or $v=\lambda \wedge v$, that is either $\lambda \leqslant v$ or $v \leqslant \lambda$. Consequently, the set of fuzzy m-bi ideals of $M$ becomes totally ordered.

We have shown in the above theorems that every m-bi ideals of $M$ is semiprime iff every fuzzy $m$ bi ideals of $M$ is semiprime. However, the following examples show that if every $m$-bi ideals of $M$ is prime (strongly prime), then it is not necessarily true that every fuzzy m-bi ideals of $M$ is prime (strongly prime).

Example 3.7. Consider the semigroup $M=\{\alpha, \beta, \gamma, \delta\}$ with the binary operation $\cdot$ given in the following table.

\begin{tabular}{|c|c|c|c|c|}
\hline$\cdot$ & $\alpha$ & $\beta$ & $\gamma$ & $\delta$ \\
\hline$\alpha$ & $\alpha$ & $\alpha$ & $\alpha$ & $\alpha$ \\
\hline$\beta$ & $\alpha$ & $\beta$ & $\gamma$ & $\beta$ \\
\hline$\gamma$ & $\alpha$ & $\gamma$ & $\beta$ & $\gamma$ \\
\hline$\delta$ & $\alpha$ & $\beta$ & $\gamma$ & $\beta$ \\
\hline
\end{tabular}

Taking $m=2$, we get, $M^{2}=\{\alpha, \beta, \gamma\}$.

Remark 3.8.

1. The 2-bi ideals in $M$ are $\{\alpha\},\{\alpha, \beta\},\{\alpha, \gamma\},\{\alpha, \beta, \gamma\}$ and $\{\alpha, \beta, \gamma, \delta\}$. Since $M$ is $m$-regular and $m$ intraregular, every fuzzy $m$-bi ideals is semiprime (see Theorem 3.2). So, all 2-bi ideals are also semiprime 2-bi ideals of $M$.

2. The prime 2-bi ideals of $M$ are $\{\alpha\},\{\alpha, \beta\},\{\alpha, \gamma\}$ and $\{\alpha, \beta, \gamma, \delta\}$. These are also strongly prime except the prime 2-bi ideal, $\{\alpha\}$, which is not strongly prime 2-bi ideal because $\{\alpha, \beta\}\{\alpha, \gamma\} \cap\{\alpha, \gamma\}\{\alpha, \beta\}=$ $\{\alpha, \beta\} \cap\{\alpha, \gamma\}=\{\alpha\}$, but none of $\{\alpha, \beta\}$ and $\{\alpha, \gamma\}$ is contained in $\{\alpha\}$.

3. $\{\alpha, \beta\}$ and $\{\alpha, \gamma\}$ are not bi-ideals of $M$, but $\{\alpha, \beta, \gamma\}$ is a bi-ideals of $M$.

4. Every fuzzy $m$-subset of $M$ is a fuzzy m-subsemigroup of $M$.

5. A fuzzy m-subset $\mu$ of $M$ is a fuzzy m-bi ideal of $M$ iff $\mu(\alpha) \geqslant \mu(x) \forall x \in M^{m}$.

Proof. Since $\mu(\alpha)=\mu(x \alpha x) \geqslant \mu(x) \wedge \mu(x)=\mu(x), \forall, x \in M^{m}$. This gives that $\mu(\alpha) \geqslant \mu(x), \forall$, $x \in M^{m}$.

Conversely, suppose that $\mu(\alpha) \geqslant \mu(x), \forall x \in M^{m}$. Then we get $x y z=x$ for $x, y, z \in\{\beta, \gamma\}$. $x y z=\alpha$ if either of $x, y$ or $z$ is $\alpha . \mu(x y z) \geqslant \mu(x) \wedge \mu(z)$. That is, $\mu$ is fuzzy $m$-bi ideal of $M$.

6. We take the fuzzy $m$-bi ideals $\mu, \lambda, \nu$ of $M$ defined by their values as $\mu(\alpha)=0.7, \mu(\beta)=0.6$, $\mu(\gamma)=0.4, \lambda(\alpha)=1, \lambda(\beta)=0.5, \lambda(\gamma)=0.4, v(\alpha)=0.7, v(\beta)=0.65, v(\gamma)=0.3$, and $\lambda \circ v(\alpha)=0.7$, $\lambda \circ \nu(\beta)=0.5, \lambda \circ v(\gamma)=0.4$. Thus $\lambda \circ \nu \leqslant \mu$ but neither $\lambda \leqslant \mu$ nor $\nu \leqslant \mu$. Hence $\mu$ is not a prime fuzzy $m$-bi ideals of $M$.

Example 3.9. Consider the semigroup $M=\{\alpha, \beta, \gamma, \delta\}$ with its representation given in the Table below:

\begin{tabular}{|l|l|l|l|l|}
\hline$\cdot$ & $\alpha$ & $\beta$ & $\gamma$ & $\delta$ \\
\hline$\alpha$ & $\alpha$ & $\alpha$ & $\alpha$ & $\alpha$ \\
\hline$\beta$ & $\alpha$ & $\beta$ & $\beta$ & $\beta$ \\
\hline$\gamma$ & $\alpha$ & $\beta$ & $\gamma$ & $\gamma$ \\
\hline$\delta$ & $\alpha$ & $\beta$ & $\gamma$ & $\gamma$ \\
\hline
\end{tabular}

Clearly, $M$ is neither regular and nor intraregular. But it is both 2-regular and 2- intraregular. 2-bi ideals are $\{\alpha\},\{\alpha, \gamma\}, M^{2}=\{\alpha, \beta, \gamma\}$ and $M$. All 2-bi ideals are strongly prime. 
Remark 3.10.

1. Each fuzzy m-subset of $M$ is its fuzzy $m$-subsemigroup.

2. A fuzzy m-subset $\mu$ of $M$ is its fuzzy m-bi ideal iff $\mu(\alpha) \geqslant \mu(\beta) \geqslant \mu(\gamma) \forall x \in M^{m}$.

Proof. Since $\mu(\alpha)=\mu(x \alpha x) \geqslant \mu(x) \wedge \mu(x)=\mu(x), \forall, x \in M^{m}$, this gives that $\mu(\alpha) \geqslant \mu(x), \forall, x \in M^{m}$. Also, $\mu(\beta)=\mu(\gamma \beta \gamma) \geqslant \mu(\gamma) \wedge \mu(\gamma)=\mu(\gamma)$, i.e., $\mu(\beta) \geqslant \mu(\gamma)$. Consequently, $\mu(\alpha) \geqslant \mu(\beta) \geqslant \mu(\gamma)$. Conversely, suppose that $\mu(\alpha) \geqslant \mu(\beta) \geqslant \mu(\gamma)$. Obviously, $\mu$ is a fuzzy m-subsemigroup of $M$. Also $u v w=\gamma$ if $u, v, w \in\{\gamma, \beta\}$ and one of these is $\gamma$ and $u v w=\beta$ if $u=v=w=\beta$. So $\mu(u v w) \geqslant \mu(u) \wedge \mu(w)$. Eventually, $\mu$ is a fuzzy m-bi ideal of $M$.

3. We consider the fuzzy $m$-bi ideals $\mu, \lambda$ and $v$ of $M$ defined by $\mu(\alpha)=0.7, \mu(\beta)=0.6, \mu(\gamma)=0.5$, $\lambda(\alpha)=1, \lambda(\beta)=0.5, \lambda(\gamma)=0.4, v(\alpha)=0.7, v(\beta)=0.65, v(\gamma)=0.3$. Then $\lambda \circ v(\alpha)=v \circ \lambda(\alpha)=0.7$, $\lambda \circ v(\beta)=v \circ \lambda(\beta)=0.5, \lambda \circ v(\gamma)=v \circ \lambda(\gamma)=0.3$. Thus $\lambda \circ v \wedge v \circ \lambda \leqslant \mu$ but neither $\lambda \leqslant \mu$ nor $\nu \leqslant \mu$. Hence $\mu$ is not strongly prime fuzzy m-bi ideals of $M$.

The following example demonstrates when the m-bi ideals and the subsets of a semigroup are also the prime and semiprime m-bi ideals.

Example 3.11. In a right zero semigroup $M$ with the cardinality $|M|>1$, we have $y x=x \forall x, y \in M$. So, for an arbitrary $x \in M, x x=x$, i.e., $x$ is idempotent, $M^{2}=M$. That is, every subset is $m$-bi ideal of $M$. Then $M$ is both m-regular and m-intraregular. Every fuzzy m-subset of $M$ is a fuzzy $m$-bi ideal of $M$. If $\mu$ is a fuzzy m-subset of $M, \mu(x y)=\mu(x) \leqslant \mu(x) \wedge \mu(y), \forall x, y \in M^{m}$. Moreover, $\mu(x y z)=\mu(x) \leqslant \mu(x) \wedge \mu(z)$, $\forall x, y, z \in M$ [12]. This implies that $\mu$ is a fuzzy $m$-bi ideal of $M$. Since $M$ is both m-regular and $m$-intraregular, every fuzzy $m$-subset of $M$ is a fuzzy semiprime $m$-bi ideal of $M$.

\section{Conclusions}

Concepts of the prime, strongly prime, and semiprime m-bi ideals, alongside their essential properties, were presented. These concepts can be interpreted in the context of some other classes of semigroups and semiring theory. These results can be utilized in different fields of soft computing such as artificial intelligence, automata theory, machine learning, and decision-making.

\section{Acknowledgment}

Our special thanks goes to anonymous refrees whose guidance made this work published.

\section{References}

[1] J. Ahsan, R. M. Latif, M. Shabir, Fuzzy quasi-ideals in semigroups, J. Fuzzy Math., 9 (2001), 259-270. 1

[2] J. S. Golan, The theory of semirings with applications in mathematics and theoretical computer science, John Wiley \& Sons, New York, (1992). 1

[3] J. M. Howie, An introduction to semigroup theory, Academic Press, New York-London, (1976). 1

[4] N. Kausar, Characterizations of non associative ordered semigroups by the properties of their fuzzy ideals with thresholds ( $\alpha, \beta]$, Prikl. Diskretn. Mat., 43 (2019), 37-59. 1

[5] N. Kausar, A. Meshari, Salahuddin, Anti fuzzy interior ideals on Ordered AG-groupoids, Eur. J. Pure Appl. Math., 13 (2020), 113-129. 1

[6] N. Kausar, A. Meshari Alesemi, Salahuddin, M. Munir, Characterizations of non-associative ordered semigroups by their intuitionistic fuzzy bi-ideals, Disc. Nonl. Compl., 9 (2020), 257-275. 1

[7] N. Kuroki, On fuzzy ideals and fuzzy bi-ideals in semigroups, Fuzzy Sets and Systems, 5 (1981), 203-215. 3.2

[8] J. N. Mordeson, D. S. Malik, N. Kuroki, Fuzzy semigroups, Springer-Verlag, Berlin, (2012). 1

[9] J. N. Mordeson, P. S. Nair, Fuzzy Graphs and Fuzzy Hypergraphs, Physica-Verlag, Heidelberg, (2000). 1

[10] M. Munir, On m-bi ideals in semigroups, Bull. Int. Math. Virtual Inst., 8 (2018), 461-467. 1, 3

[11] M. M. K. Rao, Fuzzy bi-interior ideals of semigroups, Asia Pacific. J. Math., 5 (2018), 208-218. 1

[12] M. Shabir, Y. B. Jun, M. Bano, On prime fuzzy bi-ideals of semigroups, Iran. J. Fuzzy Syst., 7 (2010), 115-128. 2, 2, 3.3, $3.4,3.5,3.6,3.11$ 
[13] T. Shah, N. Kausar, Characterizations of non-associative ordered semigroups by their fuzzy bi-ideals, Theoret. Comput. Sci., 529 (2014), 96-110. 1

[14] L. A. Zadeh, Fuzzy set, Information and Control, 8 (1965), 338-353. 1

[15] H. Zimmermann, Fuzzy sets theory and its application, Kluwer Academic Publ., New York, (2001). 1 